\title{
Treatment of stage I non-small cell lung cancer: What's trending?
}

Timothy L. McMurry, PhD, ${ }^{a}$ Puja M. Shah, MD, MS, ${ }^{b}$ Pamela Samson, MD, MPH,

Clifford G. Robinson, MD, ${ }^{\mathrm{d}}$ and Benjamin D. Kozower, MD, $\mathrm{MPH}^{\mathrm{e}}$

\section{ABSTRACT}

Objectives: Stage I non-small cell lung cancer traditionally is treated with lobectomy. Sublobar resection and stereotactic body radiation therapy provide alternative treatments for higher-risk groups. The purpose of this study was to determine the national treatment trends for stage I lung cancer.

Methods: The National Cancer Database was queried for patients with clinical stage I non-small cell lung cancer between 1998 and 2012. Patients were compared across treatment groups, and trends in treatment and disease were evaluated over the 15 -year time period.

Results: The National Cancer Database contained 369,931 patients with clinical stage I non-small cell lung cancer. After removing patients who received chemotherapy as a first course of treatment and patients with pathologic stage IV, 357,490 patients were analyzed. The first recorded cases of stereotactic body radiation therapy are in 2003 and rapidly increased to 6.6\% (2063) of all patients treated in 2012. The number of diagnoses of stage I non-small cell lung cancer steadily increased over the 15 -year period, whereas the rate of lobectomy decreased from $55 \%$ in 1998 to $50 \%$ in $2012(P<.001)$. Most of the decrease in lobectomy can be explained by the increase in the rate of sublobar resection from $12 \%$ to $17 \%(P<.001)$. The percentage of untreated patients remained stable at approximately $7 \%(P=.283)$.

Conclusions: Although the number of stage I non-small cell lung cancer cases continues to increase, lobectomy rates are decreasing while sublobar resection and stereotactic body radiation therapy rates are increasing. Although the increasing popularity of alternative therapies to lobectomy for treatment of stage I non-small cell lung cancer should allow more patients to undergo treatment, we did not observe this trend in the data. (J Thorac Cardiovasc Surg 2017;154:1080-7)

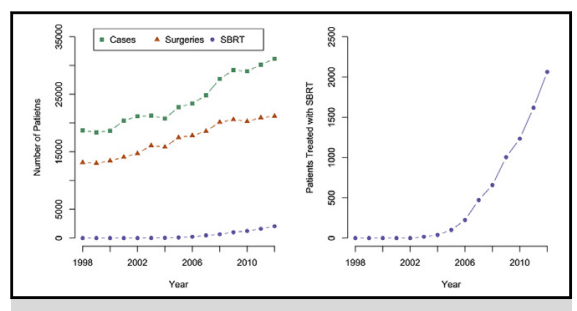

SBRT was introduced in 2003, and its use has been increasing exponentially.

\section{Central Message}

The rates of lobectomy and pneumonectomy for patients with stage I NSCLC have decreased, whereas SLR and SBRT have become more common.

\section{Perspective}

The results of this study demonstrate that the rate of surgery, particularly lobectomy, has decreased from 1998 to 2012, whereas the rates of SLR and stereotactic body radiation have increased and offset the decrease in lobectomy. Untreated patients encompass $7 \%$ of the cohort and have remained unchanged over the 15 -year study period.

See Editorial Commentary page 1088.

See Editorial page 1072 .
From the Departments of ${ }^{\mathrm{a}}$ Public Health and ${ }^{\mathrm{b}}$ Surgery, University of Virginia, Charlottesville, Va; Departments of ${ }^{\mathrm{c}}$ Surgery and ${ }^{\mathrm{d}}$ Radiation Oncology, and ${ }^{\mathrm{e}}$ Division of Cardiothoracic Surgery, Washington University, St Louis, Mo.

This work was supported by the National Institutes of Health Grant Surgical Oncology T32 CA163177 and through a Patient-Centered Outcomes Research Institute Program Award (CE-1306-00727).

Read at the 96th Annual Meeting of The American Association for Thoracic Surgery, Baltimore, Maryland, May 14-18, 2016.

Received for publication May 5, 2016; revisions received March 8, 2017; accepted for publication March 25, 2017; available ahead of print May 2, 2017.

Address for reprints: Benjamin D. Kozower, MD, MPH, Division of Cardiothoracic Surgery, Washington University, School of Medicine, One Barnes-Jewish Hospital Plaza, Suite 3108 Queeny Tower, St Louis, MO 63110 (E-mail: kozowerb@ wudosis.wustl.edu).

$0022-5223 / \$ 36.00$

Copyright (c) 2017 by The American Association for Thoracic Surgery

http://dx.doi.org/10.1016/j.jtcvs.2017.03.122
The gold standard for treatment of stage I non-small cell lung cancer (NSCLC) is lobectomy and systematic mediastinal lymph node dissection (MLND). ${ }^{1}$ Reported rates of survival after surgical resection for stage I NSCLC vary from $60 \%$ to $80 \%$ in the literature. ${ }^{1-3}$ Unfortunately, up to $25 \%$ of these "resectable" patients are not offered

Scanning this QR code will take you to the article title page. 


\section{Abbreviations and Acronyms \\ MLND = mediastinal lymph node dissection \\ NCDB $=$ National Cancer Database \\ NSCLC $=$ non-small cell lung cancer \\ SBRT = stereotactic body radiation therapy \\ SLR = sublobar resection}

lobectomy because of medical comorbidities, precluding safe resection. ${ }^{4}$ These high-risk patients subsequently may be offered a variety of other treatment options, including sublobar resection (SLR) (wedge resection or segmentectomy), radiofrequency ablation, or stereotactic body radiotherapy (SBRT). Not surprisingly, high-risk patients treated with these alternative therapies have lower 5-year survival reported in the literature. ${ }^{5,6}$ Of note, the definition of "medically inoperable" or "high-risk" patients is not well delineated.

The purpose of this study was to describe national treatment and disease trends for clinical stage I NSCLC over the 15-year period from 1998 to 2012 using a large national cancer database. We hypothesized that the frequency of SBRT had increased over this time frame and that the percentage of patients receiving no treatment had decreased because of alternative therapies available for "higherrisk" patients.

\section{MATERIALS AND METHODS}

The National Cancer Database (NCDB) is a clinical oncology database from more than 1500 facilities accredited by the Commission On Cancer. It is estimated that $70 \%$ of all malignancies in the United States are captured by the NCDB. Deidentified data were obtained from the NCDB Participant User File for all patients with NSCLC between the years 1998 and 2012. Exemption was obtained from the University of Virginia Institutional Review Board because all of the data are deidentified and contain no protected health information.

Patients with reported clinical stage I NSCLC were eligible for inclusion in the current study. Patients were excluded if they did not have clinical stage I or they received chemotherapy as a first course of treatment. Patients were further excluded if they were coded as pathologic stage IV.

Patients with stage I NSCLC were further stratified on the basis of first treatment received: surgical or nonsurgical. Surgical treatment options were categorized as lobectomy, SLR (wedge resection or segmentectomy), or pneumonectomy. Nonsurgical treatments included stereotactic body radiation therapy (SBRT), conventional radiation, and no treatment. Patients were coded as having received SBRT if the treatment modality was indicated to be stereotactic radiosurgery, the regional radiation dose was between 3000 and 10,000 cGy, and the course of radiation ran at most 14 days. Patients were coded as having received conventional radiation if they were indicated to have received radiation with treatment modality other than stereotactic radiosurgery, they received a regional dose between 5000 and $10,000 \mathrm{cGy}$, and their course of treatment lasted 21 to 120 days. Patients with unknown treatment order, with missing data for whether or not treatment was given, or who received radiation or surgery (eg, local treatment) not conforming to the described categories were placed in an "unknown/other" category.

Variables that were abstracted for this study include age, race, sex, Charlson-Deyo Comorbidity Score (coded as 0 , 1, or 2), insurance status
(Medicaid, Medicare, private, and unknown/uninsured), tumor histology (adenocarcinoma, large cell/other, and squamous cell cancers), and size in millimeters. Demographic and clinical information are shown for the overall cohort. In addition, we present time trends for treatment, tumor size, histology, regional nodes examined, and rates of pathologic upstaging.

Continuous variable comparisons were made using the Kruskal-Wallis test and are described as means or medians, as appropriate. Trends in continuous variables were assessed using weighted least squares. Categoric variables were analyzed using the chi-square test and the chi-square test for trend. Trends are illustrated using time series plots showing treatment rates by year. Analyses were performed using SAS 9.4 (SAS Institute Inc, Cary, NC) and R 3.3.2 (R Core Team, Vienna, Austria).

\section{RESULTS \\ Clinical Staging Information}

Between 1998 and 2012, the NCDB captured 1,478,751 patients with a diagnosis of NSCLC. Some $25 \%$ of these patients $(369,931)$ were diagnosed with clinical stage 1 , and the remainder had stages $2(5 \%), 3(21 \%), 4(34 \%)$, and missing $(15 \%)$.

Of the 369,931 patients with clinical stage 1 NSCLC, we excluded 11,318 who received chemotherapy as a first treatment and 1123 who were ultimately pathologically upstaged to stage IV NSCLC, leaving 357,490 patients included in the analysis.

\section{Patient Demographics and Treatment Modalities}

Patient demographics overall and divided by first treatment are shown in Table 1. Patients had a median age of 70 years (interquartile range, 63-77), $48 \%$ were male, and $90 \%$ were white. Some $53 \%$ of patients had a Charlson score of 0 , the median tumor size was $24 \mathrm{~mm}, 57 \%$ of patients were diagnosed with adenocarcinoma, and $67 \%$ of patients were insured by Medicare. Some $55 \%$ of patients received a lobectomy, $72 \%$ of patients received a surgical treatment, $6 \%$ received conventional radiation or SBRT, $7 \%$ of patients received no treatment at all, and $15 \%$ were placed in the unknown/other category. The majority of these patients $(12 \%)$ were indicated to have received some form of treatment, mostly radiation, that did not conform to our definitions.

Men were more likely to receive pneumonectomy, whereas the surgery groups, particularly the lobectomy and pneumonectomy groups, tended to be younger. Treatment patterns did differ by race $(P<.001)$, because whites were less likely than blacks to receive no treatment.

Patients with squamous cancers were more likely to receive pneumonectomies. In addition, the patients undergoing pneumonectomy had the largest tumors. Adenocarcinoma was relatively more likely to be treated with lobectomy or SLR, whereas squamous and large cell cancers had increased rates of patients treated with conventional radiation or SBRT and were relatively more likely to go untreated. 
TABLE 1. Stage 1 patient demographics and disease characteristics overall and split by first treatment

\begin{tabular}{|c|c|c|c|c|}
\hline & All patients & Lobectomy & SLR & Pneumonectomy \\
\hline $\mathrm{n}$ & 357,490 & $194,873(54.5 \%)$ & $55,769(15.6 \%)$ & $6664(1.9 \%)$ \\
\hline Male: $\mathrm{n}(\%)$ & $172,324(48.2)$ & $92,740(47.6)$ & $24,701(44.3)$ & $4343(65.2)$ \\
\hline Age: median (IQR) & $70(63-77)$ & $69(61-75)$ & $71(64-77)$ & $65(57-72)$ \\
\hline Race-shite: n (\%) & $318,919(89.2)$ & $174,384(89.5)$ & $50,480(90.5)$ & $6079(91.2)$ \\
\hline Black & 28,405 (7.9) & $14,429(7.4)$ & $3878(7.0)$ & $446(6.7)$ \\
\hline Asian/PI & $5518(1.5)$ & $3542(1.8)$ & $686(1.2)$ & $75(1.1)$ \\
\hline Other & $4648(1.3)$ & $2518(1.3)$ & $725(1.3)$ & $64(1.0)$ \\
\hline Insurance-Medicare: n (\%) & $230,289(67.1)$ & $116,518(62.0)$ & $37,444(69.5)$ & $3323(52.7)$ \\
\hline Private/other & $100,981(29.4)$ & $64,645(34.4)$ & $14,660(27.2)$ & $2658(42.1)$ \\
\hline Medicaid & $12,173(3.5)$ & $6654(3.5)$ & $1757(3.3)$ & $330(5.2)$ \\
\hline Uninsured/unknown & $0(0.0)$ & $0(0.0)$ & $0(0.0)$ & $0(0.0)$ \\
\hline Charlson-Deyo comorbidity score-0: $\mathrm{n}(\%)$ & $138,776(53.3)$ & $74,213(52.8)$ & $20,936(47.4)$ & $2127(52.6)$ \\
\hline 1 & $85,880(33.0)$ & $48,984(34.8)$ & $16,396(37.1)$ & $1438(35.6)$ \\
\hline $2+$ & $35,545(13.7)$ & $17,490(12.4)$ & $6881(15.6)$ & $477(11.8)$ \\
\hline Tumor size (mm): median (IQR) & $24(16-34)$ & $25(17-35)$ & $18(13-25)$ & $35(25-50)$ \\
\hline Histology-adenocarcinoma: $\mathrm{n}(\%)$ & $204,395(57.2)$ & $124,769(64.0)$ & $35,218(63.1)$ & $2534(38.0)$ \\
\hline Squamous & $113,572(31.8)$ & $55,633(28.5)$ & $15,488(27.8)$ & $3586(53.8)$ \\
\hline Large cell/other & $39,523(11.1)$ & 14,471 (7.4) & $5063(9.1)$ & $544(8.2)$ \\
\hline
\end{tabular}

$S L R$, Sublobar resection; SBRT, stereotactic body radiation therapy; $I Q R$, interquartile range; $P I$, Pacific Islander.

We further looked at why nonsurgical patients did not receive surgery. A reason is provided for approximately $25 \%$ of this cohort. Some $16 \%$ were contraindicated because of patient risk factors, $0.3 \%$ died before the operation could be performed, $2 \%$ had no specific reason, $4 \%$ refused, and 5\% had surgery recommended, but it was unknown if it was performed. It is unclear why the remaining approximately $75 \%$ were not treated with surgery.

\section{Trends in Stage I Diagnosis Rates}

Diagnosis rates for stage 1 NSCLC were not constant over the study period. From 1998 to $2000,26 \%$ of cases were clinical stage 1 each year. From 2001 to 2007, the rate was approximately $23 \%$. In 2008 , the rate increased to $26 \%$ and then to $28 \%$ by 2012 .

Over the 15-year study period, there is a clear trend toward identifying smaller cancers. Mean tumor size decreased from 28.2 to $23.6 \mathrm{~mm}(P<.001)$, whereas the median, 25th, and 75th percentiles show similar decreases (Figure 1).

Histology trends are shown in Table 2. From 1998 to 2000 , approximately $58 \%$ of stage 1 cancers were diagnosed as adenocarcinoma. This rate decreased to $55 \%$ between 2001 and 2005, and then increased to $60 \%$ by 2012. There is a dramatic shift starting at approximately 2001 from squamous diagnoses to large cell diagnoses.

\section{Trends in Treatment}

Table 3 shows trends in the rates of surgical treatment both by number of cases and percentage. Although the number of lobectomies increased by approximately $50 \%$, the rate of lobectomies decreased from $55 \%$ to $50 \%$
$(P<.001)$ of all patients with stage 1 . The number of pneumonectomies decreased by approximately $50 \%$, and the rate of pneumonectomies decreased from $3.4 \%$ to $1.1 \%$ of all patients $(P<.001)$. These decreases were offset by a significant increase in the number and rate of SLRs (from $12 \%$ to $17 \%$ ) and in radiation treatments. Significant use of radiation begins at approximately 2003 , when $6 \%$ of patients were treated with conventional radiation. SBRT use also is first identified in 2003. As SBRT became more frequent (Figure 2), use of conventional radiation decreased, although by 2012 the 2 types of radiation combined were used for approximately $10 \%$ of patients.

\section{Trends in Surgery}

There is a strong trend toward examination of more regional lymph nodes (Table 4 ). The median lobectomy in patients was 5 nodes examined in 1998, compared with 8 in 2012. The other 2 surgical groups showed similar increases, although patients receiving SLRs had fewer nodes examined, with a median of 0 in 1998 increasing to a median of 2 in 2012. Patients undergoing pneumonectomy had a median of 8 lymph nodes examined in 1998, increasing to 11 in 2012. As might be expected, this additional focus on regional lymph nodes has led to dramatic increases in the rate of pathologic upstaging in the surgical population (Table 5). Although only $3 \%$ of patients undergoing lobectomy were upstaged to stage II or III in 1998, that number increased to $15 \%$ by 2012 , with particularly large increases in 2003 and in 2010. The rate of upstaging in the other 2 surgical groups increased by a similar factor, and in $2012,43 \%$ of pneumonectomy cases were upstaged ( $P$ values for the trends in all 3 groups <.001). 
TABLE 1. Continued

\begin{tabular}{cccc}
\hline Conventional radiation & SBRT & None & Unknown/other \\
\hline $13,131(3.7 \%)$ & $7430(2.1 \%)$ & $26,498(7.4 \%)$ & $53,125(14.9 \%)$ \\
$6657(50.7)$ & $3429(46.2)$ & $13,493(50.9)$ & $26,961(50.8)$ \\
$75(68-81)$ & $75(69-81)$ & $75(67-81)$ & $73(66-80)$ \\
$11,688(89.0)$ & $6714(90.4)$ & $22,577(85.2)$ & $46,997(88.5)$ \\
$1173(8.9)$ & $522(7.0)$ & $3126(11.8)$ & $4831(9.1)$ \\
$128(1.0)$ & $63(0.8)$ & $390(1.5)$ & $634(1.2)$ \\
$142(1.1)$ & $131(1.8)$ & $405(1.5)$ & $663(1.2)$ \\
$10,310(80.4)$ & $5863(80.2)$ & $19,238(76.7)$ & $37,593(74.8)$ \\
$2091(16.3)$ & $1245(17.0)$ & $4765(19.0)$ & $10,917(21.7)$ \\
$427(3.3)$ & $198(2.7)$ & $1064(4.2)$ & $1743(3.5)$ \\
$0(0.0)$ & $0(0.0)$ & $0(0.0)$ & $0(0.0)$ \\
$7107(57.2)$ & $4457(60.0)$ & $11,123(57.5)$ & $18,813(58.6)$ \\
$3450(27.8)$ & $1901(25.6)$ & $5107(26.4)$ & $8604(26.8)$ \\
$1862(15.0)$ & $1072(14.4)$ & $3101(16.0)$ & $4662(14.5)$ \\
$27(20-37)$ & $21(15-29)$ & $27(20-39)$ & $27(19,38)$ \\
$4574(34.8)$ & $3445(46.4)$ & $11,456(43.2)$ & $22,399(42.2)$ \\
$5342(40.7)$ & $2505(33.7)$ & $9403(35.5)$ & $21,615(40.7)$ \\
$3215(24.5)$ & $1480(19.9)$ & $5639(21.3)$ & $9111(17.2)$ \\
\hline
\end{tabular}

Approximately two thirds of the upstaged patients were upstaged to stage II, whereas the remaining patients were upstaged to stage III.

\section{Trends in the National Cancer Database}

The number of reporting facilities in the NCDB increased slowly from 1127 to 1998 to 1152 in 2003 . Between 2003 and 2009, there was a faster rate of increase to 1286 facilities in 2009. From that point on, the number stayed relatively constant; there are 1279 facilities represented in our 2012 data set. Cases per facility increased at a relatively constant rate from 16.6 in 1998 to 24.4 in 2012.

\section{DISCUSSION}

In this study, we evaluated national trends for stage I NSCLC using a large, national database representing

\section{Tumor Sizes by Year}

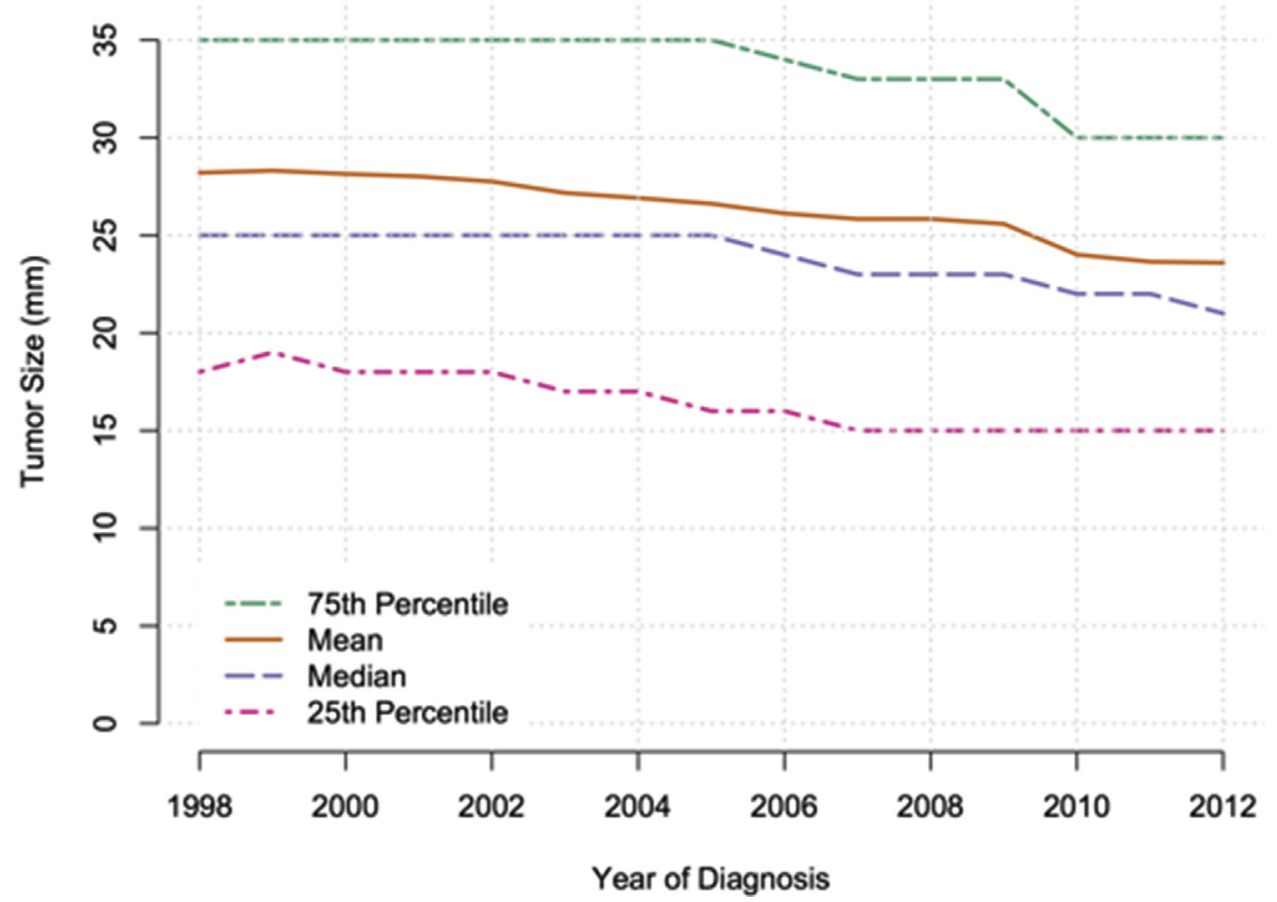

FIGURE 1. Trends in tumor size. Mean, median, 25th percentile, and 75th percentile tumor sizes (millimeters) for patients with stage I by year of diagnosis. 
more than $70 \%$ of lung cancer diagnoses in the United States. We demonstrate that the incidence of clinical stage I NSCLC represents $25 \%$ of newly diagnosed NSCLC, similar to rates quoted in the literature. ${ }^{7}$ Between 1998 and $2012,13 \%$ of patients underwent SBRT, conventional radiation, or no treatment. El-Sherif and colleagues ${ }^{8}$ quote a rate of $20 \%$ unresectability, which is consistent with our results. We hypothesized that the rate of SBRT has been increasing over the last decade, which was supported by our findings. The overall rate of surgery for stage I NSCLC decreased slightly over the 15 -year study period from $70 \%$ to $68 \%(P<.001)$, but this masks a significant shift from lobectomies to SLRs. The rate of lobectomy decreased from $55 \%$ to $50 \%$, meaning that there has been a significant decrease in the proportion of patients receiving the gold standard management option.

The increasing rate of SLR as a treatment method may be explained by several studies demonstrating relative efficacy for 5-year survival when comparing lobectomy and SLR for peripheral stage I NSCLC. SLR confers the benefit of preserved pulmonary function in patients with intrinsic lung disease or low pulmonary reserve. ${ }^{2,8}$ Landreneau and colleagues $^{9}$ demonstrated equivalent 5 -year survival for patients undergoing video-assisted thoracoscopic surgery wedge resection when compared with patients undergoing lobectomy. ${ }^{9}$ More recently, a systematic review performed by De Zoysa and colleagues ${ }^{10}$ similarly demonstrated equivalent survival for patients undergoing limited resection versus lobectomy. El-Sherif and colleagues ${ }^{11}$ showed similar results after adjusting for age and extent of node sampling. However, Landreneau and colleagues ${ }^{9}$ and De Zoysa and colleagues ${ }^{10}$ demonstrated increased locoregional recurrence with sublobar resection when compared with lobectomy. The increasing percentage of SLR and declining rate of lobectomy may be explained by an

TABLE 2. Histology by year along with chi-square test for trend

\begin{tabular}{cccc}
\hline Year & Adenocarcinoma & Squamous & Large cell \\
\hline 1998 & $10,827(57.9 \%)$ & $7333(39.2 \%)$ & $548(2.9 \%)$ \\
1999 & $10,768(58.6 \%)$ & $7085(38.6 \%)$ & $518(2.8 \%)$ \\
2000 & $11,120(59.7 \%)$ & $6967(37.4 \%)$ & $551(3.0 \%)$ \\
2001 & $11,102(54.4 \%)$ & $6809(33.4 \%)$ & $2483(12.2 \%)$ \\
2002 & $11,526(54.4 \%)$ & $6877(32.5 \%)$ & $2775(13.1 \%)$ \\
2003 & $11,587(54.5 \%)$ & $6754(31.7 \%)$ & $2937(13.8 \%)$ \\
2004 & $11,333(54.5 \%)$ & $6501(31.3 \%)$ & $2943(14.2 \%)$ \\
2005 & $12,599(55.3 \%)$ & $7028(30.9 \%)$ & $3136(13.8 \%)$ \\
2006 & $13,096(56.0 \%)$ & $7137(30.5 \%)$ & $3155(13.5 \%)$ \\
2007 & $14,073(56.7 \%)$ & $7562(30.5 \%)$ & $3178(12.8 \%)$ \\
2008 & $15,810(57.1 \%)$ & $8507(30.7 \%)$ & $3353(12.1 \%)$ \\
2009 & $17,033(58.3 \%)$ & $8900(30.5 \%)$ & $3269(11.2 \%)$ \\
2010 & $16,910(58.3 \%)$ & $8479(29.2 \%)$ & $3610(12.4 \%)$ \\
2011 & $17,928(59.5 \%)$ & $8670(28.8 \%)$ & $3547(11.8 \%)$ \\
2012 & $18,683(59.9 \%)$ & $8963(28.8 \%)$ & $3520(11.3 \%)$ \\
\hline$P$ value & $<.001$ & $<.001$ & $<.001$ \\
\hline \multicolumn{5}{c}{}
\end{tabular}

increase of diagnoses in "high-risk patients," which would help explain both the increase in SLR and the decrease in lobectomy. However, the NCDB does not collect detailed comorbidity information, such as pulmonary function or cardiac function, which makes it difficult to isolate and analyze the reasoning behind surgical procedure type.

The rate of untreated patients remained unchanged at $7 \%$. Contrary to our hypothesis, our results suggest that the advent of SBRT may have raised the threshold for offering lobectomy rather than allowing more medically inoperable patients access to therapy with curative intent.

The results further demonstrate that once SBRT appeared as a viable treatment modality, an exponential increase in this treatment method is seen. This is not unexpected, because patients who were deemed medically inoperable previously had limited treatment options, including conventional radiation, with poor survival and high recurrence rates. ${ }^{12}$ Since the advent of SBRT, medically inoperable or high-risk patients have seen improved survival; this may make it possible to treat more patients than was historically feasible. However, the only suggestion of this possibility in our results is the declining percentage of patients receiving standard treatments.

A propensity-matched study using the NCDB to compare long-term overall survival in patients with stage I NSCLC undergoing surgery or SBRT indicates that surgical patients have improved long-term survival $(68.5 \%$ vs $46 \%){ }^{13} \mathrm{~A}$ pooled analysis of 2 open-label phase III trials attempted to help elucidate the answer to this important question. Unfortunately, both trials closed early because of slow accrual; however, the Stereotactic Ablative Radiotherapy in Stage I NSCLC Patients Who Can Undergo Lobectomy and the Trial of Either Surgery or Stereotactic Radiotherapy for Early Stage (IA) Lung Cancer studies evaluated patients with early-stage (T1-2a) neoplasms who were subsequently randomized in a 1:1 fashion to lobectomy or SBRT. A total of 58 patients were enrolled and randomized between the 2 studies, 27 to lobectomy and 31 to SBRT. The 3-year survival was $88 \%$ (95\% confidence interval, $77-100)$ in the lobectomy group and $95 \%$ (95\% confidence interval, $85-100)$ in the SBRT group $(P=.037)$. In addition, there were no differences in 3-year local, regional, or distant control or in recurrence-free survival. However, the sample size in these 2 pooled analyses is small, so meaningful interpretation of the results is difficult. ${ }^{14}$ One current and one upcoming trial may help further compare outcomes in patients undergoing surgery versus SBRT for treatment of stage I NSCLC. The Sublobar Resection Versus Stereotactic Ablative Radiotherapy for lung cancer trial will randomize high-risk patients with tumors less than $4 \mathrm{~cm}$ to sublobar resection or SBRT to evaluate 3-year overall survival. The trial is currently recruiting participants. ${ }^{15}$ Likewise, the Veterans Administration Lung Cancer 
TABLE 3. First treatment by year along with chi-square test for trend

\begin{tabular}{|c|c|c|c|c|c|c|c|}
\hline Year & Lobectomy & SLR & Pneumonectomy & Radiation & SBRT & None & Unknown/other \\
\hline 1998 & $10,292(55.0 \%)$ & $2215(11.8 \%)$ & $640(3.4 \%)$ & $181(1.0 \%)$ & $0(0.0 \%)$ & $1282(6.9 \%)$ & $4098(21.9 \%)$ \\
\hline 1999 & $10,401(56.6 \%)$ & $2034(11.1 \%)$ & $575(3.1 \%)$ & $141(0.8 \%)$ & $0(0.0 \%)$ & $1294(7.0 \%)$ & $3926(21.4 \%)$ \\
\hline 2000 & $10,689(57.4 \%)$ & $2246(12.1 \%)$ & $496(2.7 \%)$ & $100(0.5 \%)$ & $0(0.0 \%)$ & $1224(6.6 \%)$ & $3883(20.8 \%)$ \\
\hline 2001 & $11,241(55.1 \%)$ & $2383(11.7 \%)$ & $449(2.2 \%)$ & $95(0.5 \%)$ & $0(0.0 \%)$ & $1646(8.1 \%)$ & $4580(22.5 \%)$ \\
\hline 2002 & $11,563(54.6 \%)$ & $2678(12.6 \%)$ & $462(2.2 \%)$ & $195(0.9 \%)$ & $0(0.0 \%)$ & $1721(8.1 \%)$ & $4559(21.5 \%)$ \\
\hline 2003 & $12,202(57.3 \%)$ & $3382(15.9 \%)$ & $499(2.3 \%)$ & $1343(6.3 \%)$ & $16(0.1 \%)$ & $1798(8.5 \%)$ & $2038(9.6 \%)$ \\
\hline 2004 & $12,140(58.4 \%)$ & $3254(15.7 \%)$ & $438(2.1 \%)$ & $1250(6.0 \%)$ & $39(0.2 \%)$ & $1696(8.2 \%)$ & $1960(9.4 \%)$ \\
\hline 2005 & $13,280(58.3 \%)$ & $3761(16.5 \%)$ & $445(2.0 \%)$ & $1347(5.9 \%)$ & $101(0.4 \%)$ & $1664(7.3 \%)$ & $2165(9.5 \%)$ \\
\hline 2006 & $13,418(57.4 \%)$ & $3965(17.0 \%)$ & $443(1.9 \%)$ & $1395(6.0 \%)$ & $224(1.0 \%)$ & $1618(6.9 \%)$ & $2325(9.9 \%)$ \\
\hline 2007 & $13,852(55.8 \%)$ & $4317(17.4 \%)$ & $402(1.6 \%)$ & $1405(5.7 \%)$ & $472(1.9 \%)$ & $1685(6.8 \%)$ & $2680(10.8 \%)$ \\
\hline 2008 & $14,925(53.9 \%)$ & $4794(17.3 \%)$ & $428(1.5 \%)$ & $1385(5.0 \%)$ & $658(2.4 \%)$ & $2070(7.5 \%)$ & $3410(12.3 \%)$ \\
\hline 2009 & $15,303(52.4 \%)$ & $4914(16.8 \%)$ & $388(1.3 \%)$ & $1331(4.6 \%)$ & $1004(3.4 \%)$ & $2282(7.8 \%)$ & $3980(13.6 \%)$ \\
\hline 2010 & $14,896(51.4 \%)$ & $5049(17.4 \%)$ & $328(1.1 \%)$ & $1105(3.8 \%)$ & $1235(4.3 \%)$ & $2171(7.5 \%)$ & $4215(14.5 \%)$ \\
\hline 2011 & $15,236(50.5 \%)$ & $5361(17.8 \%)$ & $334(1.1 \%)$ & $955(3.2 \%)$ & $1618(5.4 \%)$ & $2125(7.0 \%)$ & $4516(15.0 \%)$ \\
\hline 2012 & $15,435(49.5 \%)$ & $5416(17.4 \%)$ & $337(1.1 \%)$ & $903(2.9 \%)$ & $2063(6.6 \%)$ & $2222(7.1 \%)$ & $4790(15.4 \%)$ \\
\hline$P$ value & $<.001$ & $<.001$ & $<.001$ & $<.001$ & $<.001$ & .283 & $<.001$ \\
\hline
\end{tabular}

$S L R$, Sublobar resection; SBRT, stereotactic body radiation therapy.

Surgery or Stereotactic Radiotherapy trial has been approved and will begin enrollment soon. ${ }^{16}$

Tumor size over the study period has decreased significantly, from 2.8 to $2.4 \mathrm{~cm}$. This is similar to findings from other studies, which have demonstrated a decrease in the tumor size detected because of increased use of computed tomography scans and lung cancer screening programs. ${ }^{17-19}$ The detection of smaller tumor sizes also may explain the increasing rates of SBRT and SLR. Tumors that would have previously been too large for these treatments are now amenable to less-invasive surgical or radiotherapy ablative procedures. The increased utility of computed tomography scans would presumably give rise to detection of NSCLC at an earlier stage; however, our results show an irregular pattern in the rate of stage I cancers. More recent studies show that intentional limited resection for smaller tumors less than $2 \mathrm{~cm}$ may have comparable recurrence rates and survival to lobectomy. ${ }^{1,17,20}$ The Cancer and Leukemia Group B 140503 study is a randomized controlled trial that is nearing its accrual goal and will directly address this question. ${ }^{21}$

The Commission on Cancer recommends removal of at least 10 regional lymph nodes for stage I and II resected NSCLC. ${ }^{22}$ Our results demonstrate that by 2012 , patients undergoing lobectomy had a median of 8 lymph nodes removed, less than the recommended number by the Commission on Cancer. As expected, patients undergoing any form of SLR have fewer nodes collected. Of note, patients undergoing lobectomy were twice as likely to be pathologically upstaged compared with patients undergoing SLR,
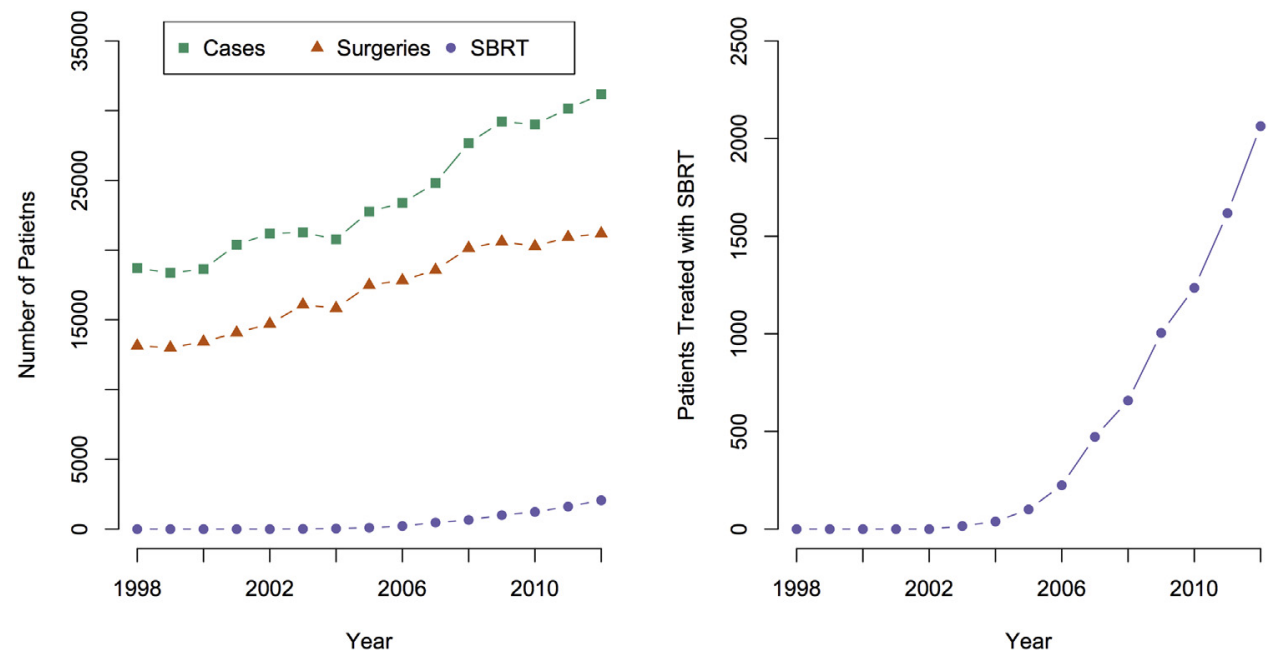

FIGURE 2. Trends in the treatment of stage I NSCLC. Annual number of stage I NSCLC cases, surgeries, and patients treated with SBRT from 1998 to 2012 (left), and the number of patients treated with SBRT with the axis rescaled to show the rapid rate of increase (right). SBRT, Stereotactic body radiation therapy. 
TABLE 4. Number of regional nodes examined (median and interquartile range) by surgery type and year

\begin{tabular}{cccc}
\hline Year & Lobectomy & Sublobar resection & Pneumonectomy \\
\hline 1998 & $5(3-9)$ & $0(0-2)$ & $8(5-14)$ \\
1999 & $6(3-10)$ & $0(0-3)$ & $9(5-15)$ \\
2000 & $6(3-10)$ & $0(0-3)$ & $8(5-13)$ \\
2001 & $6(3-10)$ & $0(0-3)$ & $10(6-14)$ \\
2002 & $6(3-10)$ & $0(0-3)$ & $9(6-15)$ \\
2003 & $6(3-10)$ & $1(0-3)$ & $9(5-15)$ \\
2004 & $6(3-10)$ & $1(0-3)$ & $10(6-15)$ \\
2005 & $7(4-11)$ & $1(0-4)$ & $10(6-15)$ \\
2006 & $7(4-11)$ & $1(0-4)$ & $10(6-15)$ \\
2007 & $7(4-11)$ & $1(0-4)$ & $10(6-15)$ \\
2008 & $7(4-11)$ & $1(0-4)$ & $12(7-17)$ \\
2009 & $7(4-12)$ & $1(0-4)$ & $11(7-17)$ \\
2010 & $7(4-12)$ & $1(0-4)$ & $12(7-17)$ \\
2011 & $8(4-12)$ & $1(0-4)$ & $11(6-17)$ \\
2012 & $8(5-12)$ & $2(0-5)$ & $11(8-17)$ \\
\hline$P$ value & $<.001$ & $<.001$ & $<.001$ \\
\hline
\end{tabular}

although this may be related to the lower lymph node sampling in SLR, leading to decreased identification of N1 or N2 disease. However, Darling and colleagues ${ }^{23}$ randomized patients with known early-stage NSCLC to MLND or mediastinal lymph node sampling. They demonstrated no difference in long-term survival or local or regional recurrence in MLND versus rigorous sampling. The authors concluded that MLND should be performed because it does not increase morbidity and it provides the most accurate staging information for adjuvant therapy planning purposes. ${ }^{23}$

TABLE 5. Pathologic upstaging by surgery type and year along with the overall percentage of patients upstaged to stage II and III by surgery type

\begin{tabular}{lccc}
\hline \multicolumn{1}{c}{ Year } & Lobectomy & Sublobar resection & Pneumonectomy \\
\hline 1998 & $3.0 \%$ & $1.6 \%$ & $11.1 \%$ \\
1999 & $3.4 \%$ & $1.6 \%$ & $13.2 \%$ \\
2000 & $3.4 \%$ & $2.0 \%$ & $14.0 \%$ \\
2001 & $3.6 \%$ & $1.7 \%$ & $16.2 \%$ \\
2002 & $4.0 \%$ & $1.9 \%$ & $13.5 \%$ \\
2003 & $6.1 \%$ & $3.4 \%$ & $19.6 \%$ \\
2004 & $5.6 \%$ & $2.8 \%$ & $17.7 \%$ \\
2005 & $5.0 \%$ & $3.0 \%$ & $18.9 \%$ \\
2006 & $5.5 \%$ & $2.8 \%$ & $16.2 \%$ \\
2007 & $6.3 \%$ & $2.7 \%$ & $22.6 \%$ \\
2008 & $10.9 \%$ & $4.9 \%$ & $32.8 \%$ \\
2009 & $11.3 \%$ & $5.9 \%$ & $33.3 \%$ \\
2010 & $15.1 \%$ & $8.3 \%$ & $45.6 \%$ \\
2011 & $14.8 \%$ & $6.4 \%$ & $46.6 \%$ \\
2012 & $15.4 \%$ & $7.1 \%$ & $43.0 \%$ \\
\hline Pathologic stage II & $5.3 \%$ & $2.4 \%$ & $14.9 \%$ \\
Pathologic stage III & $2.7 \%$ & $2.0 \%$ & $7.4 \%$ \\
\hline
\end{tabular}

\section{Study Limitations}

First, although the NCDB does capture a large majority of cancer diagnoses in the United States, it does not capture all lung cancers. Although it is the largest and most generalizable database available, there may be some specific subgroups for which these results are not applicable, such as patients in rural communities who lack adequate access to healthcare or tertiary care facilities.

Second, the NCDB captures a wide breadth of information on 30 million patients. However, the comorbidity data are not accurate and are categorized into 3 subgroups of the Charlson-Deyo score. Of note, category 1 is defined as "no comorbid conditions recorded, or no comorbid conditions present." ${ }^{24}$ Cancer registrars are well trained in abstracting the demographic and cancer-related variables, but comorbidity assessment has not been a priority. Therefore, because we cannot adequately risk adjust for treatment bias, we have not compared survival or other outcome measures between treatment groups.

Finally, the increasing number of reporting facilities and difficult to document changes in referral patterns over the 15 -year period may influence the underlying patient populations.

\section{CONCLUSIONS}

The rate of lobectomy for stage I NSCLC has declined between 1998 and 2012. Increasing use of SLR compensated for much of this decrease, although the reasons for the shifting treatment trends are unclear. Although the increasing popularity of alternatives to lobectomy for treatment of stage I NSCLC should allow more patients to undergo treatment, our results demonstrate that the percentage of untreated patients has remained stable at approximately $7 \%$ over the 15 -year study period.

\section{Conflict of Interest Statement}

Authors have nothing to disclose with regard to commercial support.

\section{References}

1. Howington JA, Blum MG, Chang AC, Balekian AA, Murthy SC. Treatment of stage I and II non-small cell lung cancer: diagnosis and management of lung cancer, 3rd ed: American College of Chest Physicians evidence-based clinical practice guidelines. Chest. 2013;143(5 Suppl):e278S-313S.

2. Jones GC, Kehrer JD, Kahn J, Koneru BN, Narayan R, Thomas TO, et al. Primary treatment options for high-risk/medically inoperable early stage NSCLC patients. Clin Lung Cancer. 2015;16:413-30.

3. Kates M, Swanson S, Wisnivesky JP. Survival following lobectomy and limited resection for the treatment of stage I non-small cell lung cancer $<=1 \mathrm{~cm}$ in size: a review of SEER data. Chest. 2011;139:491-6.

4. Donington J, Ferguson M, Mazzone P, Handy J Jr, Schuchert M, Fernando H, et al. American College of Chest Physicians and Society of Thoracic Surgeons consensus statement for evaluation and management for high-risk patients with stage I non-small cell lung cancer. Chest. 2012;142:1620-35.

5. Pennathur A, Abbas G, Christie N, Landreneau R, Luketich JD. Video assisted thoracoscopic surgery and lobectomy, sublobar resection, radiofrequency ablation, and stereotactic radiosurgery: advances and controversies in the management of early stage non-small cell lung cancer. Curr Opin Pulm Med. 2007;13:267-70. 
6. Crabtree TD, Puri V, Robinson C, Bradley J, Broderick S, Patterson GA, et al. Analysis of first recurrence and survival in patients with stage I non-small cell lung cancer treated with surgical resection or stereotactic radiation therapy. $J$ Thorac Cardiovasc Surg. 2014;147:1183-92.

7. Alberts L, El Sharouni SY, Hofman FN, Van Putte BP, Tromp E, Van Vulpen M, et al. Changes in pulmonary function after stereotactic body radiotherapy and after surgery for stage I and II non-small cell lung cancer, a description of two cohorts. Anticancer Res. 2015;35:6773-9.

8. El-Sherif A, Luketich JD, Landreneau RJ, Fernando HC. New therapeutic approaches for early stage non-small cell lung cancer. Surg Oncol. 2005;14:27-32.

9. Landreneau RJ, Sugarbaker DJ, Mack MJ, Hazelrigg SR, Luketich JD, Fetterman L, et al. Wedge resection versus lobectomy for stage I (T1 N0 M0) non-small-cell lung cancer. J Thorac Cardiovasc Surg. 1997;113:691-700.

10. De Zoysa MK, Hamed D, Routledge T, Scarci M. Is limited pulmonary resection equivalent to lobectomy for surgical management of stage I non-small-cell lung cancer? Interact Cardiovasc Thorac Surg. 2012;14:816-20.

11. El-Sherif A, Gooding WE, Santos R, Pettiford B, Ferson PF, Fernando HC, et al. Outcomes of sublobar resection versus lobectomy for stage I non-small cell lung cancer: a 13-year analysis. Ann Thorac Surg. 2006;82:408-16.

12. Kelley KD, Benninghoff DL, Stein JS, Li JZ, Byrnes RT, Potters L, et al. Medically inoperable peripheral lung cancer treated with stereotactic body radiation therapy. Radiat Oncol. 2015;10:120.

13. Puri V, Crabtree TD, Bell JM, Broderick SR, Morgensztern D, Colditz GA, et al. Treatment outcomes in stage I lung cancer: a comparison of surgery and stereotactic body radiation therapy. J Thorac Oncol. 2015;10:1776-84.

14. Chang JY, Senan S, Paul MA, Mehran RJ, Louie AV, Balter P, et al. Stereotactic ablative radiotherapy versus lobectomy for operable stage I non-small-cell lung cancer: a pooled analysis of two randomised trials. Lancet Oncol. 2015;16:630-7.

15. University of Texas Southwestern Medical Center. JoLT-Ca Sublobar Resection (SR) Versus Stereotactic Ablative Radiotherapy (SAbR) for Lung Cancer (STABLE-MATES). clinical trials.gov ID Number NCT02468024. Available at: https://clinicaltrials. gov/ct2/show/NCT02468024. Accessed April 26, 2017.

16. Moghanaki D, Karas T. Recently Approved Studies-Veterans Administration Lung Cancer Surgery Or Stereotactic Radiotherapy (VALOR). Office of
Research and Development. Available at: http://www.research.va.gov/ programs/csp/approved.cfm. Accessed April 26, 2017.

17. Reveliotis K, Kalavrouziotis G, Skevis K, Charpidou A, Trigidou R, Syrigos K. Wedge resection and segmentectomy in patients with stage I non-small cell lung carcinoma. Oncol Rev. 2014;8:234.

18. Sakurai H, Asamura H. Sublobar resection for early-stage lung cancer. Transt Lung Cancer Res. 2014;3:164-72.

19. Sroufe R, Kong F-MS. Triaging early-stage lung cancer patients into nonsurgical pathways: who, when, and what? Transl Lung Cancer Res. 2015;4: 438-47.

20. Koike T, Yamato Y, Yoshiya K, Shimoyama T, Suzuki R. Intentional limited pulmonary resection for peripheral T1 N0 M0 small-sized lung cancer. J Thorac Cardiovasc Surg. 2003;125:924-8.

21. Altorki NK, Scalzetti E, Sutherland S, Walt C. CALGB 140503 A Phase III Randomized Trial of Lobectomy versus Sublobar Resection for Small $(<=2 \mathrm{~cm})$ Peripheral Non-Small Cell Lung Cancer. Available at: https://www.calgb. org/Public/meetings/presentations/2007/cra_ws/03-140501-Altorki062007.pdf. Accessed April 26, 2017.

22. American College of Surgeons. CoC quality of care measures: $\mathrm{CoC}$ measures for quality of cancer care. Available at: https://www.facs.org/quality-programs/ cancer/ncdb/qualitymeasures. Accessed April 26, 2017.

23. Darling GE, Allen MS, Decker PA, Ballman K, Malthaner RA, Inculet RI, et al. Randomized trial of mediastinal lymph node sampling versus complete lymphadenectomy during pulmonary resection in the patient with $\mathrm{NO}$ or N1 (less than hilar) non-small cell carcinoma: results of the American College of Surgery Oncology Group Z0030 Trial. J Thorac Cardiovasc Dis. 2011; 141:662-70.

24. National Cancer Database-Data Dictionary PUF 2013. American College of Surgeons. Available at: http://ncdbpuf.facs.org/node/259. Published 2013. Accessed January 1, 2016.

Key Words: Non-small cell lung cancer, national trends, stereotactic body radiation therapy, lobectomy 